\title{
Adaptive Semiactive Cable Vibration Control: A Frequency Domain Perspective
}

\author{
Z. H. Chen ${ }^{1}$ and Y. Q. Ni ${ }^{2}$ \\ ${ }^{1}$ College of Civil Engineering, Fuzhou University, Fuzhou 350116, China \\ ${ }^{2}$ Department of Civil and Environmental Engineering, The Hong Kong Polytechnic University, Hung Hom, Kowloon, Hong Kong \\ Correspondence should be addressed to Z. H. Chen; zhchen@fzu.edu.cn
}

Received 24 March 2017; Accepted 11 July 2017; Published 10 August 2017

Academic Editor: Tai Thai

Copyright () 2017 Z. H. Chen and Y. Q. Ni. This is an open access article distributed under the Creative Commons Attribution License, which permits unrestricted use, distribution, and reproduction in any medium, provided the original work is properly cited.

\begin{abstract}
An adaptive solution to semiactive control of cable vibration is formulated by extending the linear quadratic Gaussian (LQG) control from time domain to frequency domain. Frequency shaping is introduced via the frequency dependent weights in the cost function to address the control effectiveness and robustness. The Hilbert-Huang transform (HHT) technique is further synthesized for online tuning of the controller gain adaptively to track the cable vibration evolution, which also obviates the iterative optimal gain selection for the trade-off between control performance and energy in the conventional time domain LQG (T-LQG) control. The developed adaptive frequency-shaped LQG (AF-LQG) control is realized by collocated self-sensing magnetorheological (MR) dampers considering the nonlinear damper dynamics for force tracking control. Performance of the AF-LQG control is numerically validated on a bridge cable transversely attached with a self-sensing MR damper. The results demonstrate the adaptivity in gain tuning of the AF-LQG control to target for the dominant cable mode for vibration energy dissipation, as well as its enhanced control efficacy over the optimal passive MR damping control and the T-LQG control for different excitation modes and damper locations.
\end{abstract}

\section{Introduction}

The free span of modern cable-stayed bridges increases to exceed $1000 \mathrm{~m}$, such as the Stonecutter Bridge [1], the Sutong Bridge $[1,2]$, and the Russky Bridge [2], which results in stay cables of over $500 \mathrm{~m}$ in length. Due to the extremely low inherent damping, stay cables are vulnerable to largeamplitude vibration induced by combined rain-wind excitation, parametric excitation, or other wind induced conditions [3]. For example, incidences of excessive cable vibration have been reported in the Dongting Lake Bridge in China [4], the Fred Hartman Bridge in USA [5], and the Alamillo Bridge in Spain [6].

In order to protect stay cables against the vibration mechanisms, one direct and efficient solution is to increase their structural damping by installing mechanical dampers transversely to the cables close to the deck anchorages. Passive viscous dampers are widely used by optimally tuning their damper coefficients to achieve maximum damping at only one targeted mode of cable vibration and properly selecting the damper installation location [7-11]. However, the passive dampers may potentially lead to insufficient damping to other concerned modes without increasing the damper installation location that may undesirably affect the esthetics of the bridge $[12,13]$, especially for very long cables. Alternative promising solution to cable vibration mitigation is semiactive control based on controllable magnetorheological (MR) dampers due to their real-time adjustable damping effect, fail-safe behavior, and superior energy dissipation over passive dampers $[2,6,14-25]$.

Semiactive cable damping systems rely on the control methodology in computing semiactive command force to make full use of the controllability of MR dampers. The optimal control methodology has been adopted in the semiactive cable damping system, based on either the linear quadratic regulator (LQR) control with available full state 
feedback $[24,25]$ or the linear quadratic Gaussian (LQG) control with state observation from noncollocated or collocated measurements [15-21]. The LQR or LQG control problem is usually treated from a time domain perspective by considering that the cable system model and its associated parameters are known. However, the control performance and robustness may be affected by system uncertainties, such as simplified or neglected nonlinearity, inaccurate model parameters, unmodeled dynamics, or model truncation. On the other hand, external excitations exerted on cables are usually unknown or unpredictable to be included in the control system design, so that they are usually ignored in solving the LQR or LQG control problem [26]. Since not only the intensity of external loading but also its dominant frequency affects the behavior of a controlled structure, it is of great interest in performing control design in the frequency domain for achieving the structural safety. Furthermore, the time domain LQG (denoted as T-LQG) control system generally has a constant controller gain computed with constant weight parameters that are selected by trial and error. As a result, it cannot adapt to the variation of dynamic loading and structural vibration state. For example, it was observed that the dominant mode of wind-rain-induced cable vibration may change with the vibration evolution [4].

Therefore, this paper aims at presenting an adaptive control methodology for smart cable damping from the frequency domain perspective. To achieve this goal, the LQG control is extended from the time domain to the frequency domain by synthesizing it with a frequency-shaped quadratic cost function [27]. This synthesis enables the accommodations of system uncertainties, model errors, and unknown disturbances into the controller design, which guarantees stability and robustness of the designed controller at each frequency of interest. Further, the frequency-shaped LQG controller is developed to possess adaptivity in online tuning the frequency dependent weights and the controller gain based on the Hilbert-Huang transform (HHT) technique [28] to adapt to the vibration evolution. This development improves the frequency targeting capability of the controller and obviates the iterative procedure of weight selection in the T-LQG control design. The adaptive frequency-shaped LQG (denoted as AF-LQG) control solution is implemented with a collocated sensor-damper configuration, which is realized by a self-sensing MR damper to guarantee the stability and economic efficiency of the control system [21,29]. Simulations of a stay cable equipped with the self-sensing MR damper are conducted to demonstrate the control performance of the developed control strategy, which is also compared with that achieved by using the passive MR damping control and the T-LQG control.

\section{Controlled Cable Dynamics with a Self- Sensing MR Damper}

An inclined cable transversely attached with a self-sensing MR damper near the cable anchorage on the bridge deck is considered. Assuming the profile of the cable to be shallow and using the static equilibrium configuration of the cable approximated by a parabolic curve [30], the inplane transverse motion of the cable $v(x, t)$ is derived as the following equation of motion:

$$
\begin{gathered}
m \frac{\partial^{2} v}{\partial t^{2}}+c \frac{\partial v}{\partial t}-H_{1} \frac{\partial^{2} v}{\partial x^{2}}+\left(\frac{m g \cos \alpha}{T_{1}}\right)^{2} \frac{E A}{L_{e}} \int_{0}^{L} v d x \\
=f_{e}(x, t)+F_{d}(t) \delta\left(x-x_{d}\right),
\end{gathered}
$$

where $m$ is the cable mass per unit length, $c$ is the in-plane internal cable damping, $T_{1}$ is the longitudinal component of the static cable tension, $g$ is the gravitational acceleration, $\alpha$ is the inclination angle of the cable, $E$ is the elastic modulus, $A$ is the cross-sectional area of the cable, $L$ is the cable length, $L_{e}=L\left[1+\left(m g L \cos \alpha / H_{1}\right)^{2} / 8\right]$ is the stretched length of the cable in static equilibrium state, $f_{e}(x, t)$ is the external excitation force, $\delta(\cdot)$ is the Dirac delta function, $x_{d}$ denotes the position of the damper on the cable, and $F_{d}(t)$ is the damping force component with time emulated by the forward dynamic model of the self-sensing MR damper based on the Bayesian NARX network [29, 31].

A solution of the cable motion to the partially differential equation (1) can be computed using a finite series approximation

$$
v(x, t)=\sum_{i=1}^{n} \varphi_{i}(x) q_{i}(t)
$$

where $n$ is the mode number considered, $q_{i}(t)$ is the $i$ th modal displacement, and $\varphi_{i}(x)=\sin i \pi(x / L)$ is the assumed shape function of the $i$ th mode. According to the Galerkin method, substitution of (2) into (1) results in the matrix equation

$$
\mathbf{M} \ddot{\mathbf{q}}(t)+\mathbf{C} \dot{\mathbf{q}}(t)+\mathbf{K q}(t)=\mathbf{F}_{e}(t)+\frac{2 \boldsymbol{\varphi}_{d}}{m L} F_{d}(t),
$$

where $\mathbf{q}(t)$ is the modal displacement vector, $\mathbf{M}, \mathbf{C}$, and $\mathbf{K}$ are the mass, damping, and stiffness matrix, respectively, $\mathbf{F}_{e}(t)$ is the excitation force vector, and $\varphi_{d}=\left[\varphi_{1}\left(x_{d}\right), \ldots, \varphi_{n}\left(x_{d}\right)\right]^{T}$.

For control design, (3) can be equivalently written in state-space form as

$$
\begin{aligned}
\dot{\mathbf{X}}(t) & =\mathbf{A} \mathbf{X}(t)+\mathbf{B}_{1} F_{d}(t)+\mathbf{B}_{2} \mathbf{F}_{e}(t) \\
v_{d}(t) & =\mathbf{D}_{d} \mathbf{X}(t)+w(t) \\
v_{m}(t) & =\mathbf{D}_{m} \mathbf{X}(t)+w(t)
\end{aligned}
$$

with

$$
\begin{aligned}
& \mathbf{X}=\left[\begin{array}{c}
\mathbf{q} \\
\dot{\mathbf{q}}
\end{array}\right], \\
& \mathbf{A}=\left[\begin{array}{cc}
\mathbf{0} & \mathbf{I} \\
-\mathbf{M}^{-1} \mathbf{K} & -\mathbf{M}^{-1} \mathbf{C}
\end{array}\right], \\
& \mathbf{B}_{1}=\left[\begin{array}{c}
\mathbf{0} \\
\frac{2 \mathbf{M}^{-1} \boldsymbol{\varphi}_{d}}{m L}
\end{array}\right],
\end{aligned}
$$




$$
\begin{aligned}
\mathbf{B}_{2} & =\left[\begin{array}{c}
\mathbf{0} \\
\mathbf{M}^{-1}
\end{array}\right], \\
\mathbf{D}_{d} & =\left[\begin{array}{ll}
\boldsymbol{\varphi}_{d}^{T} & \mathbf{0}
\end{array}\right], \\
\mathbf{D}_{m} & =\left[\begin{array}{ll}
\boldsymbol{\varphi}_{m}^{T} & 0
\end{array}\right],
\end{aligned}
$$

where $\boldsymbol{\varphi}_{m}=\left[\varphi_{1}\left(x_{m}\right), \ldots, \varphi_{n}\left(x_{m}\right)\right]^{T}$. The controlled output $v_{d}$ is the cable displacement at the damper attachment location $x=x_{d}$ to be used for the feedback control. The measured output $v_{m}$ is the cable displacement at the location of $x=x_{m}$ for evaluating the control performance. $w(t)$ is the measurement noise.

\section{Control Methodologies}

3.1. Time Domain $L Q G(T-L Q G)$ Control. The cable vibration control is treated as an optimal regulation problem. Based on the well-known LQR theory, a quadratic cost function in the time domain is defined as

$$
J_{t}=\int_{0}^{\infty}\left[v_{d}^{T}(t) Q_{c} v_{d}(t)+R_{c} F_{d}^{2}(t)\right] d t,
$$

where $Q_{c}$ is a positive semidefinite performance weight and $R_{c}$ is a positive weight factor for control effort penalty. Using (5), the cost function (8) can be written as

$$
J_{t}=\int_{0}^{\infty}\left[\mathbf{X}^{T}(t) \mathbf{Q X}(t)+R_{c} F_{d}^{2}(t)\right] d t,
$$

where $\mathbf{Q}=\mathbf{D}_{d}^{T} \mathbf{Q}_{c} \mathbf{D}_{d}$ is the state weight matrix. By minimizing the cost function (9) subjected to the constraints imposed by the cable-damper system dynamics in (4), the optimal control force is then determined to be

$$
F_{d}^{\mathrm{opt}}(t)=-\mathbf{G}_{c} \widehat{\mathbf{X}}(t),
$$

where $\mathbf{G}_{c}=R_{c}^{-1} \mathbf{B}_{1}^{T} \mathbf{P}$ is the controller gain and $\mathbf{P}$ satisfies the algebraic Riccati equation

$$
\mathbf{P A}+\mathbf{A}^{T} \mathbf{P}-\mathbf{P B}_{1} R_{\mathrm{c}}^{-1} \mathbf{B}_{1}^{T} \mathbf{P}+\mathbf{Q}=\mathbf{0} .
$$

$\widehat{\mathbf{X}}$ is an estimate of the system state vector $\mathbf{X}$ and is reconstructed by the Kalman filtering technique based on the measured cable displacement at the damper location

$$
\dot{\hat{\mathbf{X}}}=\mathbf{A} \widehat{\mathbf{X}}+\mathbf{B}_{1} F_{d}+\mathbf{G}_{K F}\left[v_{d}-\mathbf{D}_{d} \widehat{\mathbf{X}}\right],
$$

where $\mathbf{G}_{K F}=\mathbf{S D}_{d}^{T} R_{K F}^{-1}$ is the estimator gain and $\mathbf{S}$ is computed by the Riccati equation

$$
\mathbf{A S}+\mathbf{S A}^{T}-\mathbf{S D}_{c}^{T} R_{K F}^{-1} \mathbf{D}_{c} \mathbf{S}+\mathbf{B}_{2} \mathbf{Q}_{K F} \mathbf{B}_{2}^{T}=\mathbf{0},
$$

where $\mathbf{Q}_{K F}$ and $R_{K F}$ are the constant power spectral densities of the excitation $\mathbf{F}_{e}$ and the measurement noise $w$, respectively, with the assumption that $\mathbf{F}_{e}$ and $w$ are uncorrelated zero-mean Gaussian stochastic processes.
For the cable of slight damping, its structural energy may approximate the total of elastic and kinetic energies. By defining

$$
\mathbf{Q}=\frac{1}{2}\left[\begin{array}{ll}
\mathbf{K} & 0 \\
\mathbf{0} & \mathbf{M}
\end{array}\right]
$$

the performance index in (9) becomes the evaluation of the total cable energy. Thereby, the cable vibration reduction is achieved through the system energy control. With the decided weight matrix $\mathbf{Q}$, the control weight factor $R_{c}$ is then chosen by trial and error for the trade-off between the control force and the response reduction.

\subsection{Adaptive Frequency-Shaped LQG (AF-LQG) Control}

3.2.1. Extension of LQG Control in Frequency Domain. From the perspective of classical control, the LQR controller designed with full state feedback has certain desirable robustness properties with a guaranteed $60^{\circ}$ phase margin and $50 \%$ to infinite gain margin [32]. However, it was shown that the guaranteed robustness properties may vanish if a state estimator is introduced in the feedback loop [33]. To improve the robustness of the LQG control, Gupta [27] proposed to extend the LQG control to include a frequency-shaped cost function in terms of the frequency variable $\omega$, which is derived by applying Parseval's theorem to the time domain cost function in (8)

$$
\begin{aligned}
J_{\omega} & =\frac{1}{2 \pi} \int_{-\infty}^{\infty}\left[v_{d}^{*}(j \omega) Q_{c}(j \omega) v_{d}(j \omega)\right. \\
& \left.+F_{d}^{*}(j \omega) R_{c}(j \omega) F_{d}(j \omega)\right] d \omega,
\end{aligned}
$$

where * denotes the complex conjugate and $Q_{c}(j \omega)$ and $R_{c}(j \omega)$ are frequency dependent weight functions that are Hermitian at all frequencies. The main advantage of the frequency dependent weight functions is that they facilitate the incorporation of known and desired control system behaviors into the control synthesis process and influence the control performance and robustness by varying emphases over different frequencies of interest.

The weight functions $Q_{c}(j \omega)$ and $R_{c}(j \omega)$ are assumed to be rational functions of squared frequency $\omega^{2}$ and are decomposed, respectively, as

$$
\begin{aligned}
& Q_{c}(j \omega)=Q_{1}^{*}(j \omega) Q_{1}(j \omega) \\
& R_{c}(j \omega)=R_{1}^{*}(j \omega) R_{1}(j \omega) .
\end{aligned}
$$

Defining

$$
\begin{aligned}
& v_{d f}(j \omega)=Q_{1}(j \omega) v_{d}(j \omega) \\
& F_{d f}(j \omega)=R_{1}(j \omega) F_{d}(j \omega)
\end{aligned}
$$

the cost function (15) can be rewritten as

$$
\begin{aligned}
J_{\omega} & =\frac{1}{2 \pi} \\
& \cdot \int_{-\infty}^{\infty}\left[v_{d f}^{*}(j \omega) v_{d f}(j \omega)+F_{d f}^{*}(j \omega) F_{d f}(j \omega)\right] d \omega .
\end{aligned}
$$


If $Q_{1}(j \omega)$ and $R_{1}(j \omega)$ are proper, they can be realized by the following input-output relationships:

$$
\begin{gathered}
\dot{\boldsymbol{\eta}}(t)=\mathbf{A}_{\eta} \boldsymbol{\eta}(t)+\mathbf{B}_{\eta} Y_{d}(t) \\
v_{d f}(t)=\mathbf{C}_{\eta} \boldsymbol{\eta}(t)+D_{\eta} v_{d}(t), \\
\dot{\boldsymbol{\zeta}}(t)=\mathbf{A}_{\zeta} \boldsymbol{\zeta}(t)+\mathbf{B}_{\zeta} F_{d}(t) \\
F_{d f}(t)=\mathbf{C}_{\zeta} \boldsymbol{\zeta}(t)+D_{\zeta} F_{d}(t),
\end{gathered}
$$

where $\mathbf{A}_{i}, \mathbf{B}_{i}, \mathbf{C}_{i}$, and $D_{i}(i=\eta, \zeta)$ are the state matrix, input matrix, output matrix, and feedthrough term for the systems in (17a) and (17b).

Equations (19a) and (20a) are now augmented with (4), (5), and (6) to form an extended system model as

$$
\begin{aligned}
\dot{\widetilde{\mathbf{X}}}(t) & =\widetilde{\mathbf{A}} \widetilde{\mathbf{X}}(t)+\widetilde{\mathbf{B}}_{1} F_{d}(t)+\widetilde{\mathbf{B}}_{2} \mathbf{F}_{e}(t) \\
v_{d}(t) & =\widetilde{\mathbf{D}} \widetilde{\mathbf{X}}(t) \\
v_{m}(t) & =\widetilde{\mathbf{D}}_{m} \widetilde{\mathbf{X}}(t),
\end{aligned}
$$

where the newly constructed state vectors and matrices are given by

$$
\begin{aligned}
\widetilde{\mathbf{X}} & =\left[\begin{array}{l}
\mathbf{X} \\
\boldsymbol{\eta} \\
\zeta
\end{array}\right], \\
\widetilde{\mathbf{A}} & =\left[\begin{array}{ccc}
\mathbf{A} & \mathbf{0} & \mathbf{0} \\
\mathbf{B}_{\eta} \mathbf{D}_{d} & \mathbf{A}_{\eta} & \mathbf{0} \\
\mathbf{0} & \mathbf{0} & \mathbf{A}_{\zeta}
\end{array}\right], \\
\widetilde{\mathbf{B}}_{1} & =\left[\begin{array}{c}
\mathbf{B}_{1} \\
\mathbf{0} \\
\mathbf{B}_{\zeta}
\end{array}\right], \\
\widetilde{\mathbf{B}}_{2} & =\left[\begin{array}{c}
\mathbf{B}_{2} \\
\mathbf{0} \\
\mathbf{0}
\end{array}\right], \\
\widetilde{\mathbf{D}}_{d} & =\left[\begin{array}{lll}
\mathbf{D}_{d} & \mathbf{0} & \mathbf{0}
\end{array}\right], \\
\widetilde{\mathbf{D}}_{m} & =\left[\begin{array}{lll}
\mathbf{D}_{m} & \mathbf{0} & \mathbf{0}
\end{array}\right] .
\end{aligned}
$$

Equations (19b) and (20b) then become

$$
\begin{aligned}
& v_{d f}(t)=\widetilde{\mathbf{C}}_{1} \widetilde{\mathbf{X}}(t) \\
& F_{d f}(t)=\widetilde{\mathbf{C}}_{2} \widetilde{\mathbf{X}}(t)+D_{\zeta} F_{d}(t)
\end{aligned}
$$

with

$$
\begin{aligned}
\widetilde{\mathbf{C}}_{1} & =\left[\begin{array}{lll}
D_{\eta} \mathbf{D}_{d} & \mathbf{C}_{\eta} & \mathbf{0}
\end{array}\right], \\
\widetilde{\mathbf{C}}_{2} & =\left[\begin{array}{lll}
\mathbf{0} & \mathbf{0} & \mathbf{C}_{\zeta}
\end{array}\right] .
\end{aligned}
$$

According to the above definitions and Parseval's theorem, the cost function (18) is transformed back to the time domain as

$$
\tilde{J}_{t}=\int_{0}^{\infty}\left[v_{d f}^{T}(t) v_{d f}(t)+F_{d f}^{T}(t) F_{d f}(t)\right] d t
$$

Substitution of (23) and (24) into (26) yields the cost function in terms of the extended state vector $\widetilde{\mathbf{X}}$

$$
\begin{aligned}
& \widetilde{J}_{t} \\
& =\int_{0}^{\infty}\left[\widetilde{\mathbf{X}}^{T}(t) \widetilde{\mathbf{Q}} \widetilde{\mathbf{X}}(t)+2 \widetilde{\mathbf{X}}^{T}(t) \widetilde{\mathbf{N}} F_{d}(t)+\widetilde{R} F_{d}^{2}(t)\right] d t,
\end{aligned}
$$

where

$$
\begin{aligned}
\widetilde{\mathbf{Q}} & =\widetilde{\mathbf{C}}_{1}^{T} \widetilde{\mathbf{C}}_{1}+\widetilde{\mathbf{C}}_{2}^{T} \widetilde{\mathbf{C}}_{2}, \\
\widetilde{\mathbf{N}} & =\widetilde{\mathbf{C}}_{2}^{T} D_{\zeta}, \\
\widetilde{R} & =D_{\zeta}^{2} .
\end{aligned}
$$

Then, the control law to minimize $\widetilde{J}_{t}$ in (27) can be derived as

$$
F_{d}^{\mathrm{opt}}(t)=-\widetilde{\mathbf{G}}_{c} \widetilde{\mathbf{X}}(t)=-\widetilde{R}^{-1}\left(\widetilde{\mathbf{B}}_{1}^{T} \widetilde{\mathbf{P}}+\widetilde{\mathbf{N}}^{T}\right) \widetilde{\mathbf{X}}(t),
$$

where $\widetilde{\mathbf{P}}$ is the positive semidefinite solution to the following Riccati equation:

$$
\widetilde{\mathbf{P}} \widetilde{\mathbf{A}}+\widetilde{\mathbf{A}}^{T} \widetilde{\mathbf{P}}-\left(\widetilde{\mathbf{P}} \widetilde{\mathbf{B}}_{1}+\widetilde{\mathbf{N}}\right) \widetilde{R}^{-1}\left(\widetilde{\mathbf{P}} \widetilde{\mathbf{B}}_{1}+\widetilde{\mathbf{N}}\right)^{T}+\widetilde{\mathbf{Q}}=\mathbf{0}
$$

With the full state information estimated from (12), the active control law (29) is designed as

$$
F_{d}^{\mathrm{opt}}(t)=-\widetilde{\mathbf{G}}_{c}[\widehat{\mathbf{X}} \boldsymbol{\eta} \zeta]^{T} .
$$

3.2.2. Frequency Dependent Weight Functions. From field tests of wind-rain induced vibration of bridge cables, it has been observed that this kind of cable vibration is dominated by one of the first few in-plane modes of the cable with low frequencies $[4,5,34,35]$, and the dominant mode may change with the vibration evolution [4]. Therefore, the objective is to design a feedback controller for reducing the cable vibration at the dominant mode. To achieve this goal, the frequency dependent performance weight for the $i$ th vibration mode can be designed in the form of

$$
Q_{1}(j \omega)=\frac{\gamma_{i} \beta_{0} j \omega}{\omega_{c i}^{2}-\omega^{2}+\beta_{0} j \omega}
$$

which can be written into the equivalent state-space equation as given in (19a) and (19b) with

$$
\begin{aligned}
\mathbf{A}_{\eta} & =\left[\begin{array}{cc}
0 & 1 \\
-\omega_{c i}^{2} & -\beta_{0}
\end{array}\right], \\
\mathbf{B}_{\eta} & =\left[\begin{array}{c}
0 \\
\gamma_{i} \beta_{0}
\end{array}\right],
\end{aligned}
$$




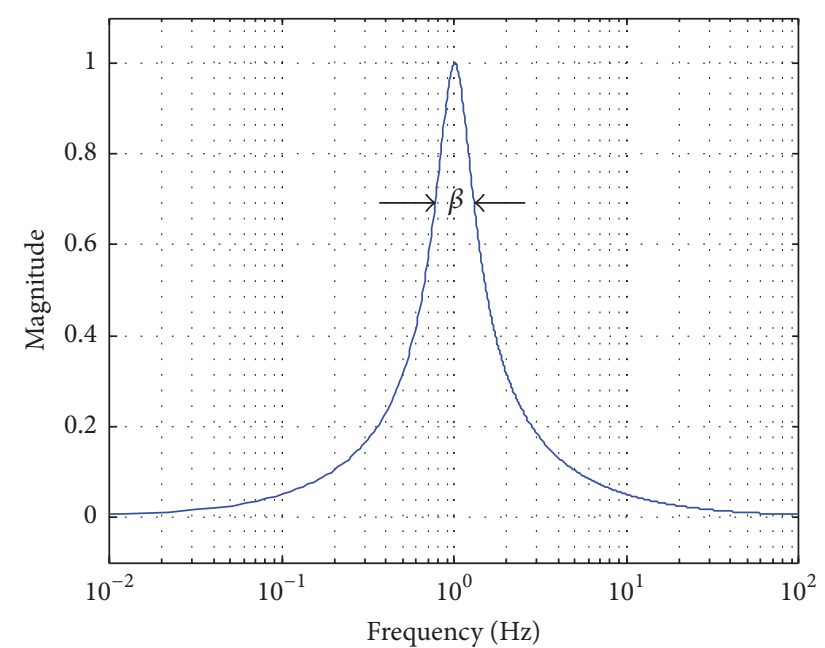

FIGURE 1: Magnitude plot of frequency response of $Q_{1 i}(j \omega)$.

$$
\begin{aligned}
\mathbf{C}_{\eta} & =\left[\begin{array}{ll}
0 & 1
\end{array}\right], \\
D_{\eta} & =0 .
\end{aligned}
$$

Equation (32) defines a second-order band-pass filter with critical frequency $\omega_{c i}$, filter bandwidth $\beta_{0}$, and filter gain $\gamma_{i}$ at the critical frequency. Figure 1 shows the magnitude plot of frequency response of $Q_{1}(j \omega)$ with $\gamma_{i}=1, \beta_{0}=$ $0.5 \mathrm{~Hz}$, and $\omega_{c i}=1 \mathrm{~Hz}$ as an example. It indicates that the cable response is selectively reduced with heavier penalty levy over a narrowband of frequencies around the critical frequency $\omega_{c i}$, which is the dominant frequency of the cable vibration. The frequency band to be punished is defined by the bandwidth $\beta_{0}$, so that the chosen performance weight in (32) is more robust to and less affected by the uncertainties in the system parameters as it depends on a band of frequency.

On the other hand, the control law (31) is designed based on the finite-dimensional cable model in (4) truncated from the continuous structure of infinite dimension. When such a finite-dimensional controller is applied to a real structure, only the modes of interest are stabilized while the stability of other residual (uncontrolled and unmodeled) modes is unassured. The interaction between the controller and the residual modes may deteriorate the control performance and sometimes induce instability, known as spillover phenomenon [36]. To reduce or eschew the negative spillover effects, the control weight function $R_{1}(j \omega)$ for the cable vibration control is selected to penalize the high frequency control energy more than the low frequency control energy. This in turn compensates for the unmodeled dynamics at high frequencies to obtain good high frequency robustness. Since $D_{\zeta}$ should not be zero in any case to guarantee the positive $\widetilde{R}$ in (28), $R_{1}(j \omega)$ needs to be a nonstrict proper function and is taken to be the form of a second-order filter

$$
R_{1}(j \omega)=\frac{\left(j \omega+\omega_{r 1}\right)^{2}}{\left(j \omega+\omega_{r 2}\right)^{2}}, \quad\left(\omega_{r 1}<\omega_{r 2}\right)
$$

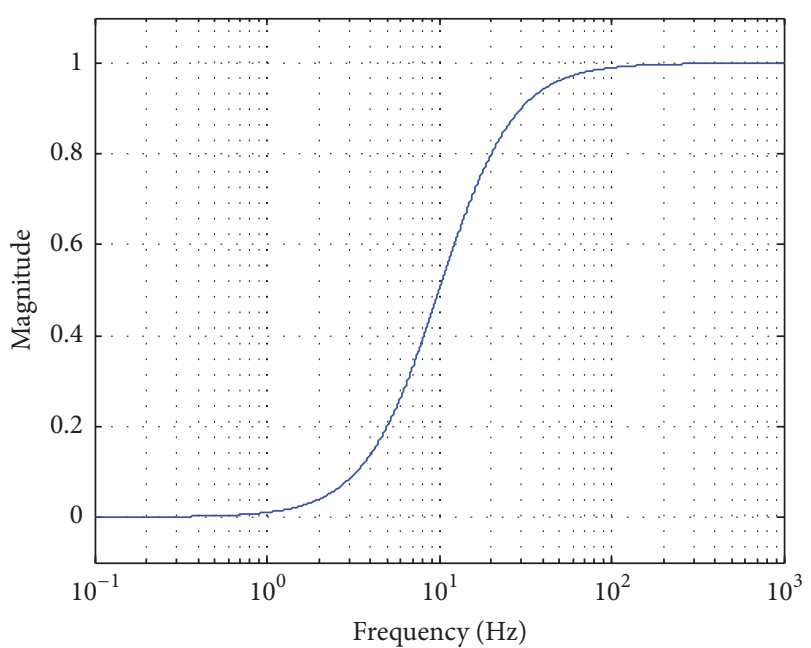

FIGURE 2: Magnitude plot of frequency response of $R_{1}(j \omega)$.

in which $\omega_{r 1}$ and $\omega_{r 2}$ are frequencies for the filter design. The equivalent state-space representation of $R_{1}(j \omega)$ is given in (20a) and (20b) with

$$
\begin{aligned}
& \mathbf{A}_{\zeta}=\left[\begin{array}{cc}
0 & 1 \\
\omega_{r 2}^{2} & -2 \omega_{r 2}
\end{array}\right], \\
& \mathbf{B}_{\zeta}=\left[\begin{array}{l}
0 \\
1
\end{array}\right], \\
& \mathbf{C}_{\zeta}=\left[\begin{array}{ll}
\omega_{r 1}^{2}-\omega_{r 2}^{2} & 1
\end{array}\right], \\
& D_{\zeta}=1 .
\end{aligned}
$$

Figure 2 illustrates the magnitude plot of frequency response of $R_{1}(j \omega)$ with $\omega_{r 1}=0.1 \mathrm{~Hz}$ and $\omega_{r 2}=10 \mathrm{~Hz}$ as an example.

3.2.3. HHT-Based Weight Self-Tuning. To accommodate the variation of dominant mode in cable vibration evolution, the frequency-shaped controller gain $\widetilde{\mathbf{G}}_{c}$ in (31) is desired to be adaptively adjusted with the mode variation by updating the frequency dependent weight function in (32). For such adaptivity, the critical frequency $\omega_{c i}$ is online tuned to be the dominant modal frequency of the cable-damper system identified from the measured cable response $v_{d}$ with the employment of the HHT technique [28].

The HHT technique is a combination of empirical mode decomposition (EMD) and Hilbert spectral analysis, in which the EMD is a key integrant to advantage the Hilbert spectral analysis. The EMD adaptively decomposes the measured response signal $v_{d}$ into a series of intrinsic mode functions (IMFs) that admit well-behaved Hilbert transform by a procedure called sifting process. At the end of the sifting process, the decomposition of the signal $v_{d}(t)$ into $n$ empirical modes is achieved as

$$
v_{d}(t)=\sum_{i=1}^{n} c_{i}(t)+r_{n}(t),
$$


where $c_{i}(t)$ is the $i$ th IMF of the measured signal and $r_{n}(t)$ is the residue. Then, the Hilbert transform is performed on each IMF component as

$$
\widetilde{c}_{i}(t)=\operatorname{HT}\left[c_{i}(t)\right]=\frac{1}{\pi} \int_{-\infty}^{+\infty} c_{i}(t) \frac{1}{t-\tau} d \tau
$$

With the Hilbert transform, the analytical signal for each IMF component is defined as

$$
z_{a i}(t)=c_{i}(t)+j \widetilde{c}_{i}(t)=a_{i}(t) \exp \left(j \theta_{i}(t)\right)
$$

with

$$
\begin{aligned}
& a_{i}(t)=\sqrt{c_{i}^{2}(t)+\widetilde{c}_{i}^{2}(t),} \\
& \theta_{i}(t)=\arctan \left(\frac{\widetilde{c}_{i}(t)}{c_{i}(t)}\right),
\end{aligned}
$$

where $a_{i}(t)$ is the instantaneous amplitude and $\theta_{i}(t)$ is the instantaneous phase. The instantaneous frequency corresponding to each IMF is given by

$$
\omega_{i}(t)=\frac{d \theta_{i}(t)}{d t} .
$$

From the frequency-time distribution of the instantaneous amplitude, designated as the Hilbert amplitude spectrum $H_{a}(\omega, t)$, the energy spectrum $H_{a}^{2}(\omega, t)$ additionally gives the instantaneous energy as

$$
I E(t)=\int_{\omega} H_{a}^{2}(\omega, t) d \omega
$$

The instantaneous energy indicates the energy fluctuation of each IMF component with time.

For cable control implementation, the frequency dependent weight function $Q_{1}(j \omega)$ is updated by shifting the critical frequency $\omega_{c i}$ to target for the dominant mode identified by the HHT; namely, $\omega_{c i}=\omega_{i}$. Meanwhile, the filter gain $\gamma_{i}$ in (32) for the $i$ th cable mode can be adjusted based on the proportion of the instantaneous energy $I E_{i}$ of the $i$ th IMF component over the total instantaneous energy of the entire set of IMFs; that is,

$$
\gamma_{i}=\frac{I E_{i}}{\sum_{i=1}^{n} I E_{i}} .
$$

The value of $\gamma_{i}$ implies the contribution of the corresponding mode to the cable vibration. Hence, heavier penalty is placed on the dominant vibration mode during the control implementation.

The HHT technique has been recognized as a powerful, local, and adaptive tool in frequency-time analysis. A great advantage of the HHT is the effective use of the data. Limited signal within a narrow time window is adequate for effectively extracting the instantaneous frequency information. This advantage results in inappreciable computation effort in identifying and updating the parameters for the frequency dependent weight and enables its synthesis in the controller for practical implementation.

3.3. Semiactive Closed-Loop Controller Synthesis. To facilitate the closed-loop control using the optimal active control force computed by (10) or (31), a semiactive constraint is introduced by taking the intrinsic dissipativity and force limitation properties of the self-sensing MR damper into account to derive the desired semiactive control force implementable by the damper

$$
F_{d}^{\mathrm{des}}(t)= \begin{cases}-\operatorname{sgn}\left(\dot{v}_{d}\right) \cdot\left|F_{\max }(t)\right|, & \text { if } F_{d}^{\mathrm{opt}}(t) \cdot \dot{v}_{d}<0,\left|F_{d}^{\mathrm{opt}}(t)\right|>\left|F_{\max }(t)\right| \\ -\operatorname{sgn}\left(\dot{v}_{d}\right) \cdot\left|F_{d}^{\mathrm{opt}}(t)\right|, & \text { if } F_{d}^{\mathrm{opt}}(t) \cdot \dot{v}_{d}<0,\left|F_{\min }(t)\right| \leq\left|F_{d}^{\mathrm{opt}}(t)\right| \leq\left|F_{\max }(t)\right| \\ -\operatorname{sgn}\left(\dot{v}_{d}\right) \cdot\left|F_{\min }(t)\right|, & \text { others, }\end{cases}
$$

where $\dot{v}_{d}=\dot{v}\left(x_{d}, t\right)$ is the velocity across the damper; $\operatorname{sgn}(\cdot)$ is the signum function; and $F_{\text {min }}(t)$ and $F_{\text {max }}(t)$ are the minimum and maximum damper forces estimated by the forward dynamic model $[29,31]$ with the instant velocity $\dot{v}_{d}$ at zero and maximum voltage levels, respectively.

To implement the desired semiactive control force, a force tracking controller based on the inverse dynamics of the self-sensing MR damper is employed to decide the voltage command for compensating the damper nonlinearity [21, 31].

\section{Numerical Studies of Cable Damping}

4.1. Cable Control Simulation System. Since its opening to traffic in the end of 2000, the cable-stayed Dongting Lake Bridge has been observed to have frequent occurrences of cable vibration under simultaneous action of rain and wind before the deployment of MR-based damping technology for cable vibration mitigation [14]. A typical cable of $121.9 \mathrm{~m}$ long in this bridge is selected for numerical verification of vibration control by installing a self-sensing MR damper at the location of $2.0 \%$ of the cable length $\left(x_{d}=0.02 L\right)$ from the deck-cable connection. Other main parameters of the cable are inclination angle $\theta=35.2^{\circ}$, cross-sectional area $A=111.2$ $\times 10^{-4} \mathrm{~m}^{2}$, mass per unit length $m=51.8 \mathrm{~kg} / \mathrm{m}$, initial tension $T=3150 \mathrm{kN}$, and elastic modulus $E=2.0 \times 10^{5} \mathrm{MPa}$. The first five in-plane modal frequencies of the cable are 1.07, 2.14, $3.20,4.23$, and $5.32 \mathrm{~Hz}$, and the corresponding first five modal damping ratios are $0.18 \%, 0.16 \%, 0.12 \%, 0.10 \%$, and $0.11 \%$.

Large-amplitude rain-wind induced oscillation of this cable has been measured in situ lasting 45 days in 2003, 
TABLE 1: Evaluation of cable damping performance.

\begin{tabular}{lcccccc}
\hline \multirow{2}{*}{ Control scheme } & \multicolumn{3}{c}{ Second-mode excitation } & \multicolumn{3}{c}{ Third-mode excitation } \\
& $v_{m}^{\text {rms }}(1 / 4 L)(\mathrm{mm})$ & $\Delta(\%)$ & $F_{d}^{\text {rms }}(\mathrm{N})$ & $v_{m}^{\text {rms }}(1 / 6 L)(\mathrm{mm})$ & $\Delta(\%)$ & $F_{d}^{\text {rms }}(\mathrm{N})$ \\
\hline Without control & 25.01 & - & - & 12.59 & - & - \\
Passive MR control & 14.50 & 42.0 & 797.37 & 7.07 & 43.9 & 776.18 \\
T-LQG control & 12.59 & 49.7 & 409.28 & 5.84 & 53.6 & 375.82 \\
AF-LQG control & 10.79 & 56.9 & 517.27 & 4.95 & 60.6 & 472.09 \\
\hline
\end{tabular}

Note. $\Delta$ denotes the percent reduction of the controlled RMS cable response over the uncontrolled case.

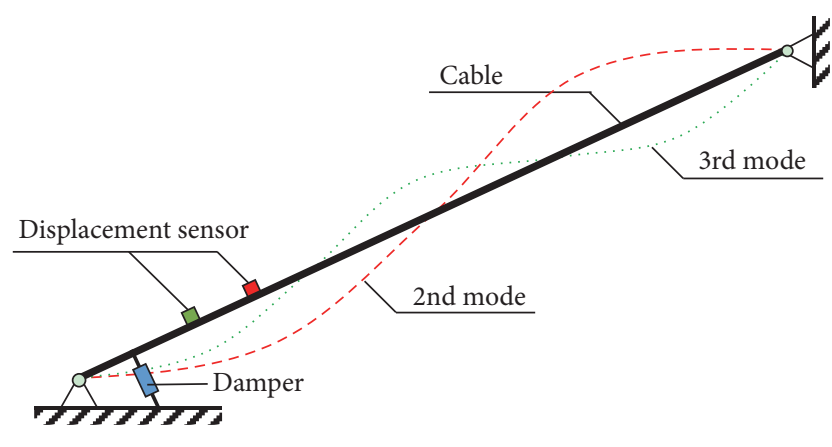

FIGURE 3: Configurations of cable-damper system.

which revealed that the overall dominant mode of cable oscillation during the observed rain-wind excitations is the third mode, accompanying other lower frequency modes [4]. In the absence of a well-established model of this excitation behavior, the cable-damper system is simulated with stochastic excitation to mainly result in the second- or third-mode response, which is given as

$$
f_{e}(x, t)=\Gamma(t) \sin \frac{i \pi x}{L}, \quad(i=2 \text { or } 3),
$$

where $\Gamma(t)$ is generated with a band-limited white noise with a peak amplitude of $5000 \mathrm{~N}$ and a frequency range of $0.5-5 \mathrm{~Hz}$. The displacement at the quarter or one-sixth of the cable span $(1 / 4 L$ or $1 / 6 L)$ from the bottom cable anchorage, where the second or third mode exhibits maximum response, is measured to evaluate the control performance. Figure 3 illustrates the configurations of the cable-MR damper system. Control simulations are conducted to examine the performance of the smart cable damping effectiveness with the self-sensing MR damper operated in a passive mode and semiactive modes with the conventional T-LQG control and the proposed AFLQG control.

4.2. Efficacy of Different Control Schemes. The control performance of the self-sensing MR damper operated in a passive mode is evaluated by commanding a spectrum of constant voltage between $0.0 \mathrm{~V}$ and $5.0 \mathrm{~V}$. An optimal voltage level is selected to minimize the root-mean-square (RMS) cable displacement $\left(v_{m}^{\mathrm{rms}}\right)$ when the cable is subjected to a single mode excitation frequency. As shown in Table 1, the passively operated MR damper activated at an optimal voltage level of $4.0 \mathrm{~V}$ and $3.5 \mathrm{~V}$ is able to reduce the uncontrolled RMS cable

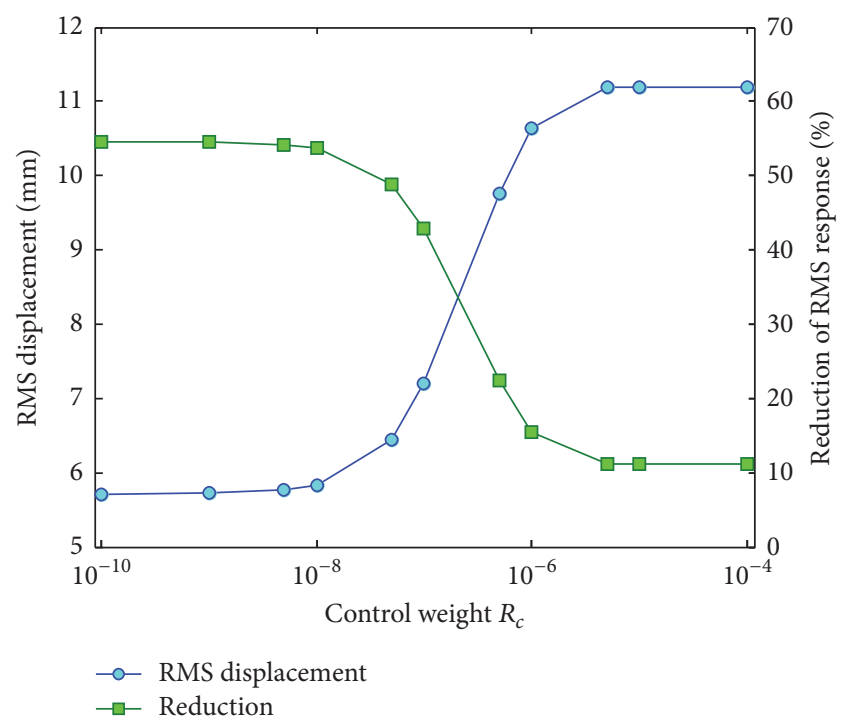

FIGURE 4: RMS cable response with different control weights in TLQG control for the third-mode excitation.

displacement by $42.0 \%$ and $43.9 \%$ for the second- and thirdmode excitations, respectively.

With the self-sensing MR damper commanded by the TLQG controller, a range of control weights $R_{c}$ are considered in evaluation of its control effectiveness. The optimal controller is determined through the trial and error that results in a minimum RMS cable response for each mode. Figures 4 and 5 illustrate the controlled cable response and the corresponding damper force under the third-mode excitation. The RMS cable displacement decreases with the increment of the control weight $R_{c}$ in the range of $\left[1 \times 10^{-8}, 5\right.$ $\left.\times 10^{-6}\right]$ that results in the reduced damper force. The response reduction becomes insignificant for $R_{c}$ being smaller than $1 \times$ $10^{-8}$, which is a result of the allowable force capacity limited by the magnetic saturation effect on the MR fluid inside the damper, as evidenced in Figure 5. In addition, due to the constraint of the residual friction force inherent in the selfsensing MR damper, the attenuation level of the RMS cable response is comparable for $R_{c}$ being larger than $5 \times 10^{-6}$. Hence, the optimal controller with $R_{c}=1 \times 10^{-8}$ is selected to achieve a 53.6\% reduction of the uncontrolled RMS response for the third-mode excitation frequency, which represents a $13.6 \%$ increase in performance beyond the optimal passive MR control. Likewise, when the cable is excited in the second 


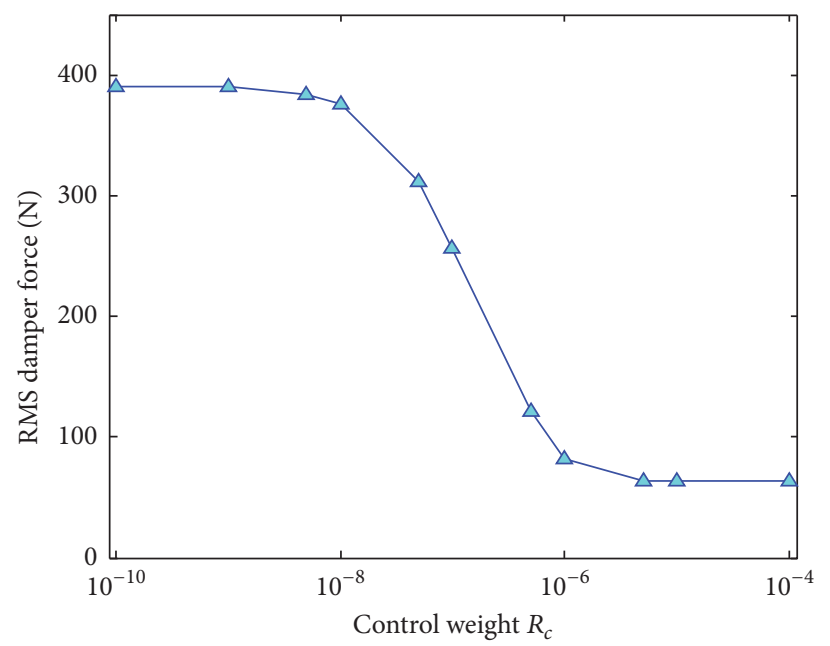

FIGURE 5: RMS damper force with different control weights in T-LQG control for the third-mode excitation.

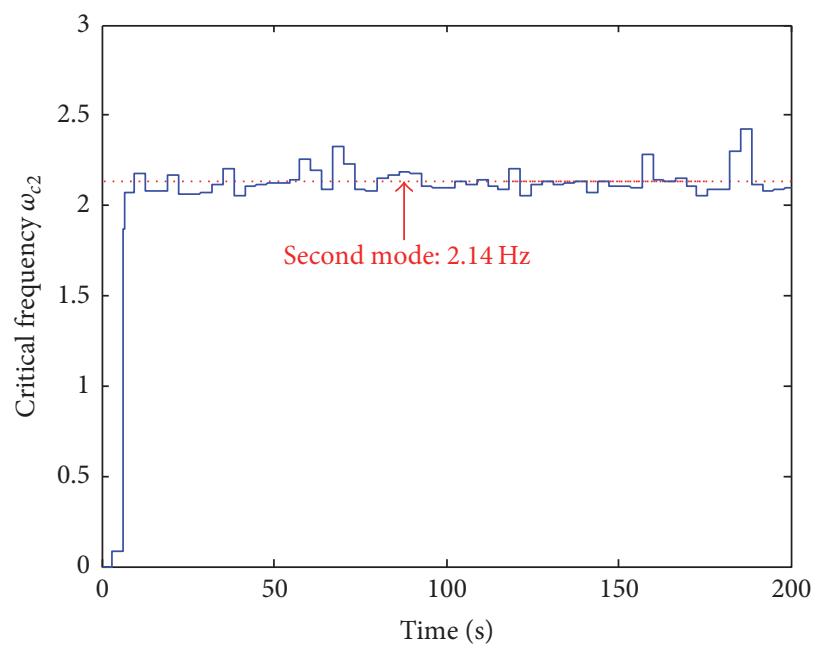

(a)

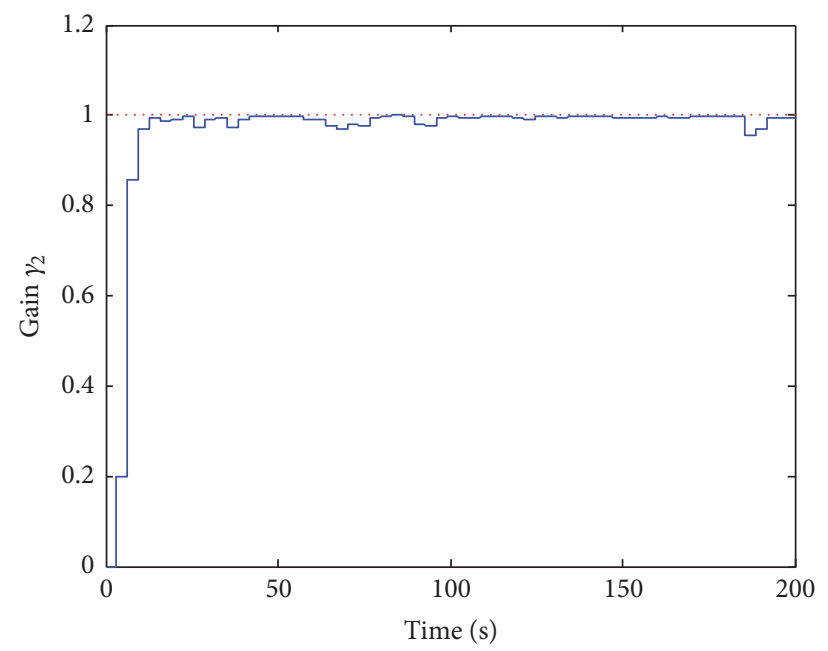

(b)

FIGURE 6: Identified information for the second-mode control: (a) critical frequency and (b) filter gain.

mode, the controlled smart damper is able to reduce the RMS cable displacement by $49.7 \%$ and $13.2 \%$ over the uncontrolled and passively controlled cases, respectively, as listed in Table 1. The improved performance in the T-LQG control requires the RMS MR damper forces of $375.82 \mathrm{~N}$ and $409.28 \mathrm{~N}$ for the second- and third-mode excitations, respectively, which are about half of the corresponding optimal passive damping forces.

With the feature of weight self-tuning in the AF-LQG control strategy, the trial and error task of finding appropriate weights in the T-LQG control is avoided. The frequency dependent weights in the simulation are taken in the forms of (32) and (34). By using the HHT technique, the IMF components are extracted through the EMD procedure from the feedback signal in a moving time window of $5.12 \mathrm{~s}$ for identifying the instantaneous frequency. Then, the critical frequency $\omega_{c i}$ of the band-pass filter in (32) is automatically tuned to be the identified dominant vibration mode. The filter gain $\gamma_{i}$ is decided based on the instantaneous energies of the IMF components according to (42). The bandwidth $\beta_{0}$ for the filter is set to be $0.5 \mathrm{~Hz}$. Hence, the system response in a neighborhood of the dominant modal frequency is penalized through the narrowband control design. The frequencies $\omega_{r 1}$ and $\omega_{r 2}$ for the control weight in (34) are taken as $0.5 \mathrm{~Hz}$ and the fifth modal frequency $5.32 \mathrm{~Hz}$, respectively, to define the filter bandwidth for weighing the high frequency control effort. Figures 6 and 7 display the identified critical frequencies and the filter gains for updating the frequency dependent weight functions in the control scenarios under the second- and third-mode excitations, respectively. It is demonstrated that the dominant cable modes are able to be identified reliably, at which the narrowband controller is targeted, while the penalty on the target mode is adjusted in an automatic fashion based on its energy intensity level. From Table 1, it is indicated that the AF-LQG controller can significantly suppress the cable vibrations excited in 


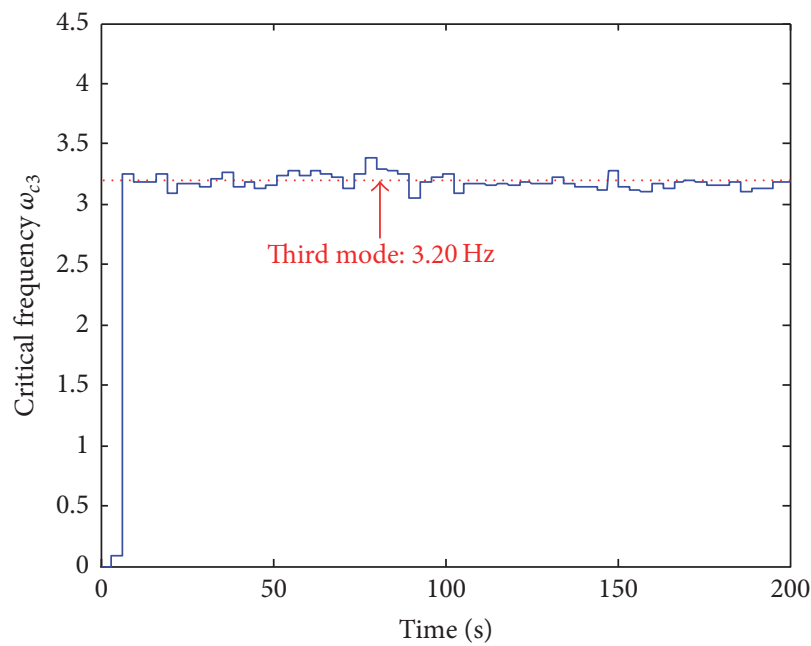

(a)

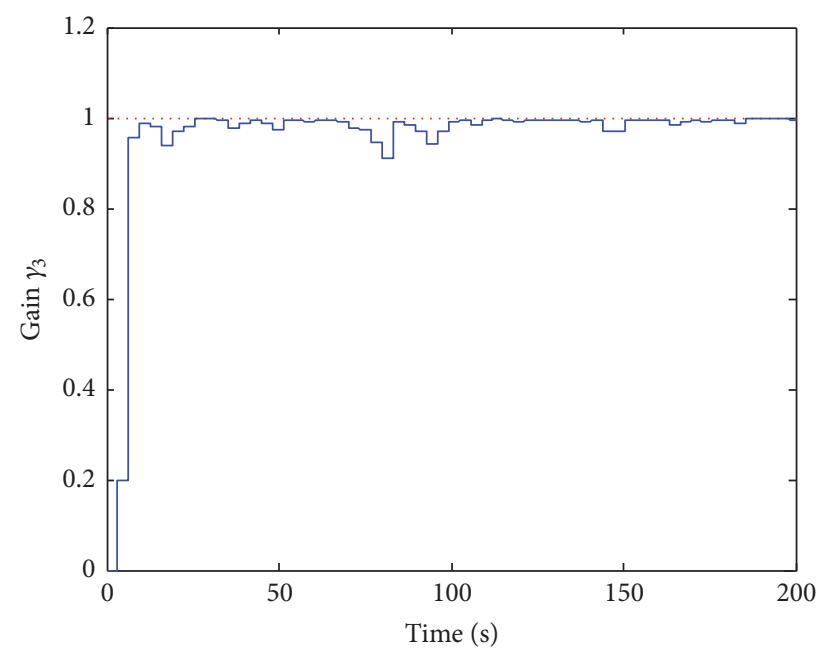

(b)

FIGURE 7: Identified information for the third-mode control: (a) critical frequency and (b) filter gain.

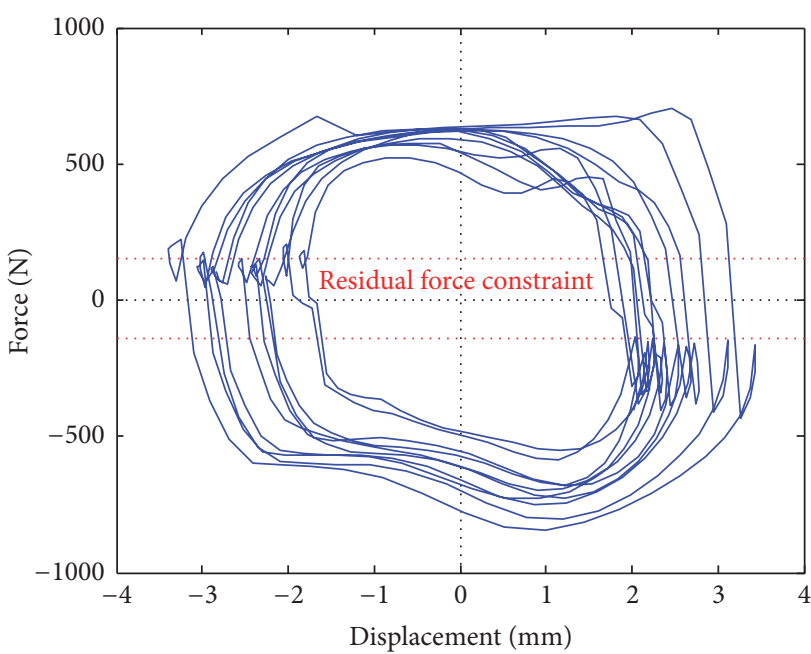

(a)

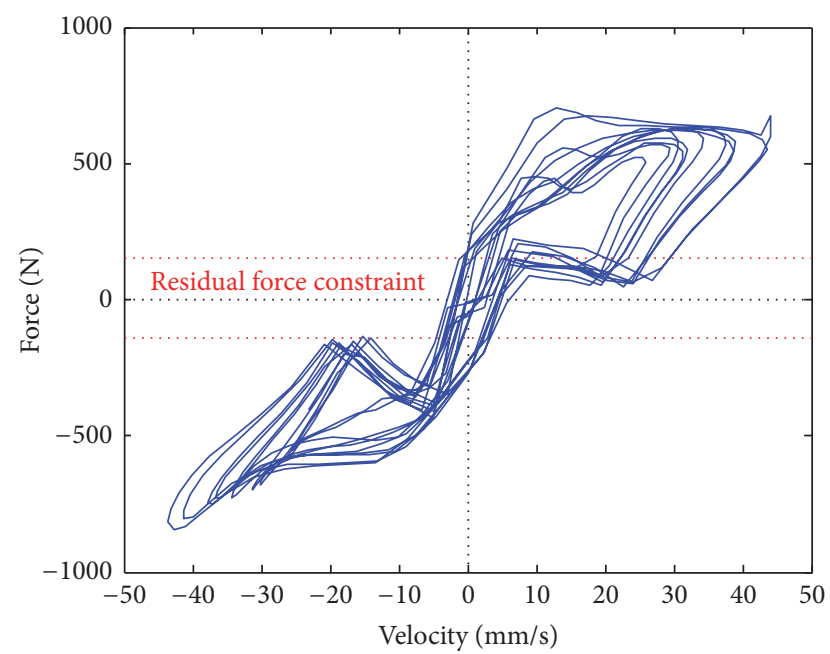

(b)

FIGURE 8: Hysteresis behaviors of self-sensing MR damper during the second-mode control: (a) force-displacement trajectory and (b) forcevelocity trajectory.

the second and third modes, which is evaluated by $56.9 \%$ and $60.6 \%$ reductions of the RMS cable responses over the uncontrolled cases, respectively. This represents $25.6 \%$ and $29.9 \%$ enhancements of the control effectiveness over the optimal passive MR control with much smaller RMS damper forces, as well as $14.3 \%$ and $15.1 \%$ improved performance beyond the T-LQG control with modest increases in RMS damper forces for the cable excited in second and third modes, respectively.

Figures 8 and 9 show the hysteretic force-displacement and force-velocity behaviors of the self-sensing MR damper (in a time window of $5 \mathrm{~s}$ ) with the AF-LQG control for the second- and third-mode excitations, respectively. It is seen that the MR damper is uncontrollable if the desired force is within the residual force constraint produced at the zero voltage level, and thereby a small portion of nondissipative force exists for lower velocity values. Furthermore, the forcedisplacement trajectories exhibit the negative stiffness behavior provided by the self-sensing MR damper. The negative stiffness force yields the reduced local stiffness of the cable at the damper position, which augments the amplitude of the damper displacement to dissipate increased amount of vibrational energy due to the improved cable damping [25] and, hence, results in overall better control performance.

4.3. Performance at Various Damper Locations. Performance of the considered passive and semiactive MR damping control strategies at different damper attachment locations is further examined under the third-mode excitation. The control results are shown in Figures 10-12. For comparison, at 


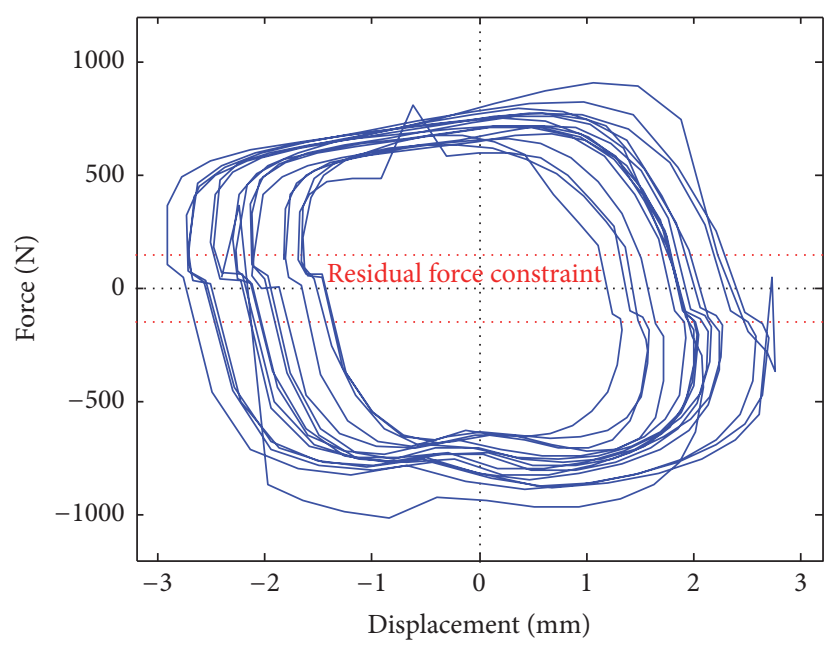

(a)

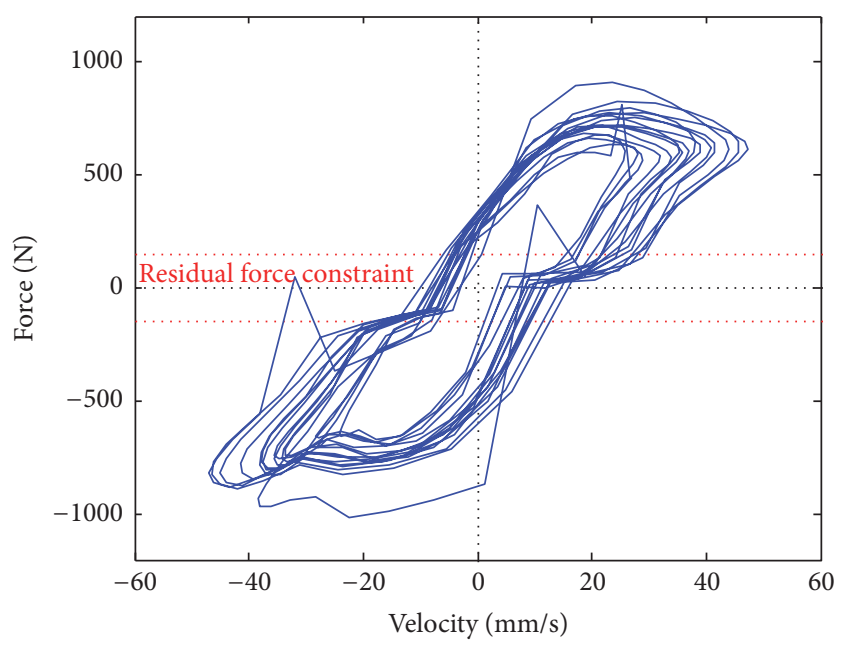

(b)

FIGURE 9: Hysteresis behaviors of self-sensing MR damper during the third-mode control: (a) force-displacement trajectory and (b) forcevelocity trajectory.

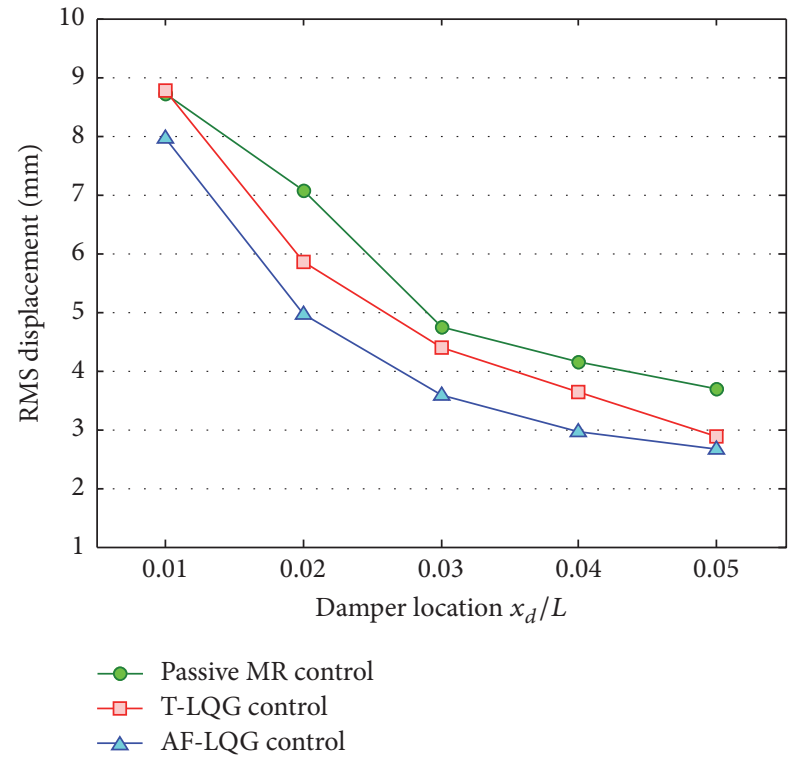

Figure 10: RMS cable responses with different control strategies and various damper locations.

each damper location, the passive MR control with an optimal constant voltage is determined over a range of voltage levels from $0.0 \mathrm{~V}$ to $5.0 \mathrm{~V}$, while the T-LQG control is chosen to result in the best semiactive damping performance by finding the corresponding control weight $R_{c}$ ranging from $1 \times 10^{-10}$ to $1 \times 10^{-4}$. It is obvious that the control effectiveness for each control strategy degrades with the attachment location $x_{d}$ of the self-sensing MR damper near the lower cable anchorage. When moving the damper position $x_{d}$ from $0.01 \mathrm{~L}$ to $0.05 \mathrm{~L}$, the RMS cable displacement can be further decreased by $57.5 \%$ for the optimal passive MR control, $67.2 \%$ for the TLQG control, and $66.7 \%$ for the AF-LQG control. The T-LQG

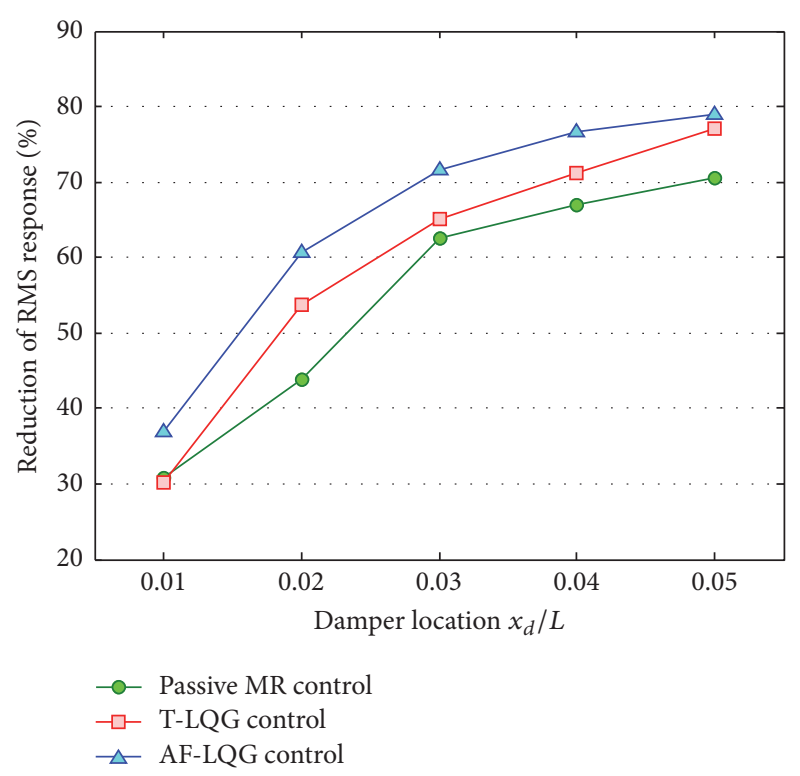

FIGURE 11: RMS response reductions over uncontrolled case with different control strategies and various damper locations.

control performs better than the optimal passive MR control, when $x_{d}$ is larger than 0.01 , with $17.4 \%, 7.4 \%, 12.5 \%$, and $22.2 \%$ further reductions of RMS cable displacement compared to the optimal passive case at the damper locations of $0.02 \mathrm{~L}$, $0.03 L, 0.04 L$, and $0.05 L$, respectively. The RMS control forces commanded in the semiactive T-LQG and AF-LQG control modes are lower than those in the passive MR damping cases as well for all the examined damper locations. Although the passively operated MR damper located at $x_{d}=0.01 \mathrm{~L}$ provides comparable cable response reduction with the $\mathrm{T}$ LQG control, the required RMS control force is about twice that of the LQG control. At each damper location, the MR 


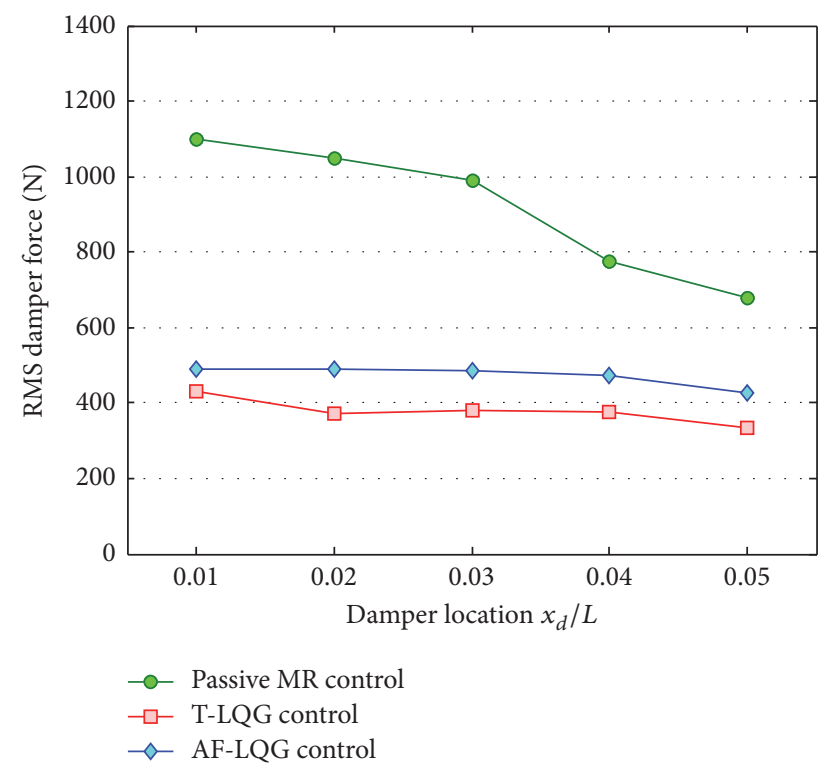

FIGURE 12: RMS damper forces for various damper locations.

damper controlled by the AF-LQG strategy always achieves superior efficacy in mitigating the RMS cable response over that controlled by the optimal passive MR or the T-LQG control, with only a modest increase in RMS damper force relative to the T-LQG control. The AF-LQG control can further improve the RMS response reduction from $8.0 \%$ to $19.0 \%$ over the T-LQG control and from $8.7 \%$ to $30.0 \%$ over the optimal passive control, with consistent levels of RMS control force, over a wide range of damper location $x_{d} / L=$ 0.01-0.05.

\section{Conclusions}

An adaptive frequency-shaped LQG (AF-LQG) control strategy is developed for effectively operating self-sensing MR dampers to suppress vibration of bridge cables. The frequency shaping introduced in the developed control strategy is advantageous to account for system uncertainties, unmodeled dynamics, and unknown disturbances, which assures the effectiveness and robustness of the control system. Adaptivity in updating the controller gain for adapting to the transient structural dynamics is realized by further incorporating the HHT technique to capture the instantaneous frequency information of the controlled cable system. Moreover, the AF-LQG scheme allows nonlinear dynamics of the selfsensing MR damper to be accommodated in the control loop. The resulting AF-LQG controller has been applied to a bridge cable installed with a self-sensing MR damper through numerical simulation, which reaches the following conclusions: (i) the developed AF-LQG control scheme can be realized by the self-sensing MR damper with the feedback information from collocated displacement and force sensors. Both the AF-LQG and T-LQG control outperform the optimized passive MR control in reducing the cable response with the required control force much lower than that in the passive case; (ii) the AF-LQG control embedded with the HHT is able to online update the controller gain adaptively with the dominant instantaneous frequency well captured and the mode penalties self-tuned, which thus improves its control efficacy over the T-LQG control with an optimally selected control weight; (iii) apparent negative stiffness behavior of the self-sensing MR damper is explored under the AF-LQG control to enhance the smart cable damping performance; and (iv) performance examinations conducted with various damper installation locations further reveal the superiority of the developed AF-LQG control over the optimal passive and T-LQG control strategies.

\section{Conflicts of Interest}

The authors declare that they have no conflicts of interest.

\section{Acknowledgments}

The authors gratefully acknowledge the support of this research by the National Natural Science Foundation of China (Grant no. 51608128), the Fujian Provincial Natural Science Foundation of China (Grant no. 2016J05123), and Education Department of Fujian Province, China (Grant no. JA15908).

\section{References}

[1] J. M. Ko and Y. Q. Ni, "Structural health monitoring and intelligent vibration control of cable-supported bridges: research and application," KSCE Journal of Civil Engineering, vol. 7, no. 6, pp. 701-716, 2003.

[2] F. Weber and H. Distl, "Amplitude and frequency independent cable damping of Sutong Bridge and Russky Bridge by MR dampers," Structural Control and Health Monitoring, vol. 22, no. 2, pp. 237-254, 2015.

[3] A. Larsen and G. L. Larose, "Dynamic wind effects on suspension and cable-stayed bridges," Journal of Sound and Vibration, vol. 334, no. 1, pp. 2-28, 2015.

[4] Y. Q. Ni, X. Y. Wang, Z. Q. Chen, and J. M. Ko, "Field observations of rain-wind-induced cable vibration in cablestayed Dongting Lake Bridge," Journal of Wind Engineering and Industrial Aerodynamics, vol. 95, no. 5, pp. 303-328, 2007.

[5] D. Zuo and N. P. Jones, "Interpretation of field observations of wind- and rain-wind-induced stay cable vibrations," Journal of Wind Engineering and Industrial Aerodynamics, vol. 98, no. 2, pp. 73-87, 2010.

[6] J. R. Casas and A. C. Aparicio, "Rain-wind-induced cable vibrations in the Alamillo cable-stayed bridge (Sevilla, Spain). Assessment and remedial action," Structure and Infrastructure Engineering, vol. 6, no. 5, pp. 549-556, 2010.

[7] B. M. Pacheco, Y. Fujino, and A. Sulekh, "Estimation curve for modal damping in stay cables with viscous damper," Journal of Structural Engineering (United States), vol. 119, no. 6, pp. 19611979, 1993.

[8] S. Krenk and S. r. Nielsen, "Vibrations of a shallow cable with a viscous damper," The Royal Society of London. Proceedings. Series A. Mathematical, Physical and Engineering Sciences, vol. 458, no. 2018, pp. 339-357, 2002. 
[9] Y. Fujino and N. Hoang, "Design formulas for damping of a stay cable with a damper," Journal of Structural Engineering, vol. 134, no. 2, pp. 269-278, 2008.

[10] S. Cheng, N. Darivandi, and F. Ghrib, “The design of an optimal viscous damper for a bridge stay cable using energy-based approach," Journal of Sound and Vibration, vol. 329, no. 22, pp. 4689-4704, 2010.

[11] L. Sun and L. Chen, "Free vibrations of a taut cable with a general viscoelastic damper modeled by fractional derivatives," Journal of Sound \& Vibration, vol. 335, pp. 19-33, 2015.

[12] X. Y. Wang, Y. Q. Ni, J. M. Ko, and Z. Q. Chen, "Optimal design of viscous dampers for multi-mode vibration control of bridge cables," Engineering Structures, vol. 27, no. 5, pp. 792-800, 2005.

[13] F. Weber, G. Feltrin, M. Maślanka, W. Fobo, and H. Distl, "Design of viscous dampers targeting multiple cable modes," Engineering Structures, vol. 31, no. 11, pp. 2797-2800, 2009.

[14] Z. Q. Chen, X. Y. Wang, J. M. Ko et al., "MR damping system for mitigating wind-rain induced vibration on Dongting Lake cable-stayed bridge," Wind and Structures, vol. 7, no. 5, pp. 293304, 2004.

[15] E. A. Johnson, R. E. Christenson, and B. F. Spencer Jr., "Semiactive damping of cables with sag," Computer-Aided Civil and Infrastructure Engineering, vol. 18, no. 2, pp. 132-146, 2003.

[16] E. A. Johnson, G. A. Baker, B. F. Spencer Jr., and Y. Fujino, "Semiactive damping of stay cables," Journal of Engineering Mechanics, vol. 133, no. 1, pp. 1-11, 2007.

[17] Y. F. Duan, Y. Q. Ni, and J. M. Ko, "State-derivative feedback control of cable vibration using semiactive magnetorheological dampers," Computer-Aided Civil and Infrastructure Engineering, vol. 20, no. 6, pp. 431-449, 2005.

[18] F. Weber, G. Feltrin, and M. Motavalli, "Measured linearquadratic-Gaussian controlled damping," Smart Materials and Structures, vol. 14, no. 6, pp. 1172-1183, 2005.

[19] R. E. Christenson, B. F. Spencer Jr., and E. A. Johnson, "Experimental verification of smart cable damping," Journal of Engineering Mechanics, vol. 132, no. 3, pp. 268-278, 2006.

[20] H. Li, M. Liu, J. Li, X. Guan, and J. Ou, "Vibration control of stay cables of the shandong binzhou yellow river highway bridge using magnetorheological fluid dampers," Journal of Bridge Engineering, vol. 12, no. 4, pp. 401-409, 2007.

[21] Z. H. Chen, K. H. Lam, and Y. Q. Ni, "Enhanced damping for bridge cables using a self-sensing MR damper," Smart Materials and Structures, vol. 25, no. 8, Article ID 085019, 2016.

[22] J. Høgsberg and S. Krenk, "Energy dissipation control of magneto-rheological damper," Probabilistic Engineering Mechanics, vol. 23, no. 2-3, pp. 188-197, 2008.

[23] H. W. Huang, L. M. Sun, and X. L. Jiang, "Vibration mitigation of stay cable using optimally tuned MR damper," Smart Structures and Systems, vol. 9, no. 1, pp. 35-53, 2012.

[24] F. Weber and C. Boston, "Clipped viscous damping with negative stiffness for semi-active cable damping," Smart Materials and Structures, vol. 20, no. 4, Article ID 045007, 2011.

[25] F. Weber and H. Distl, "Semi-active damping with negative stiffness for multi-mode cable vibration mitigation: Approximate collocated control solution," Smart Materials and Structures, vol. 24, no. 11, Article ID 115015, 2015.

[26] B. Basu and S. Nagarajaiah, "A wavelet-based time-varying adaptive LQR algorithm for structural control," Engineering Structures, vol. 30, no. 9, pp. 2470-2477, 2008.

[27] N. K. Gupta, "Frequency-shaped cost functionals: extension of linear-quadratic-Gaussian design methods," Journal of Guidance and Control, vol. 3, no. 6, pp. 529-535, 1980.
[28] N. E. Huang, Z. Shen, S. R. Long et al., "The empirical mode decomposition and the Hilbert spectrum for nonlinear and non-stationary time series analysis," The Royal Society of London. Proceedings. Series A. Mathematical, Physical and Engineering Sciences, vol. 454, no. 1971, pp. 903-995, 1998.

[29] Z. H. Chen, Y. Q. Ni, and S. W. Or, "Characterization and modeling of a self-sensing MR damper under harmonic loading," Smart Structures and Systems, vol. 15, no. 4, pp. 1103-1120, 2015.

[30] H. M. Irvine, Cable Structures, MIT Press, Cambridge, MA, USA, 1981.

[31] Y. Q. Ni, Z. H. Chen, and S. W. Or, "Experimental identification of a self-sensing magnetorheological damper using soft computing," Journal of Engineering Mechanics, vol. 141, no. 7, pp. 113, 2015.

[32] B. D. O. Anderson and D. L. Mingori, Optimal Control: Linear Quadratic Methods, Prentice Hall, Englewood Cliffs, NJ, USA, 1990.

[33] J. C. Doyle, “Guaranteed Margins for LQG Regulators," IEEE Transactions on Automatic Control, vol. 23, no. 4, pp. 756-757, 1978.

[34] J. A. Main, N. P. Jones, and H. Yamaguchi, "Characterization of rain-wind induced stay-cable vibrations from full-scale measurement," in Proceedings of the 4th International Symposium on Cable Dynamics, pp. 235-242, Montreal, Canada, April 2001.

[35] R. S. Phelan, P. P. Sarkar, and K. C. Mehta, "Full-scale measurements to investigate rain-wind induced cable-stay vibration and its mitigation," Journal of Bridge Engineering, vol. 11, no. 3, pp. 293-304, 2006.

[36] M. J. Balas, "Feedback control of flexible systems," IEEE Transactions on Automatic Control, vol. AC-23, no. 4, pp. 673-679, 1978. 


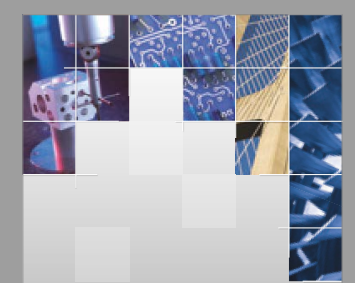

\section{Enfincering}
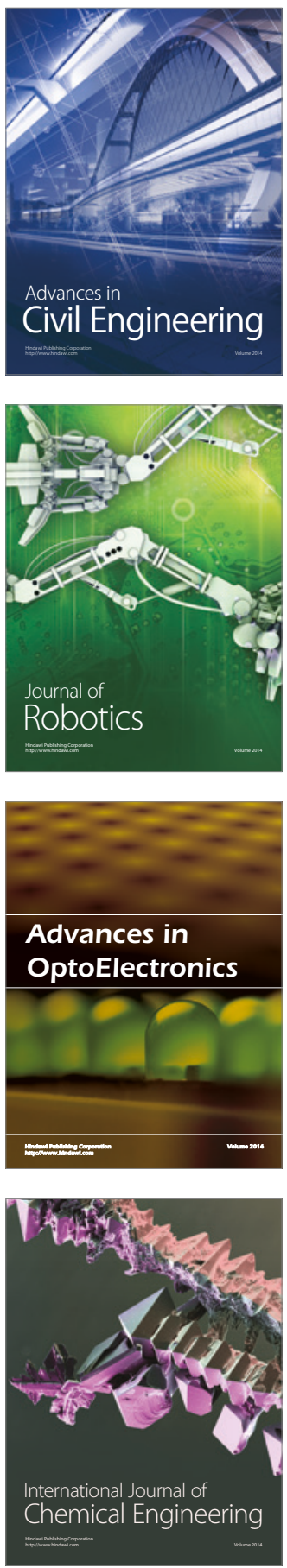

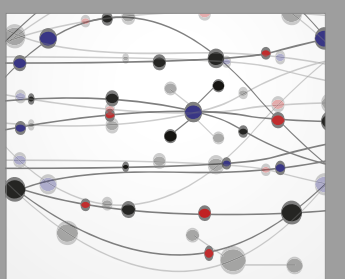

The Scientific World Journal

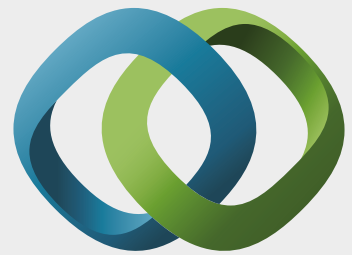

\section{Hindawi}

Submit your manuscripts at

https://www.hindawi.com
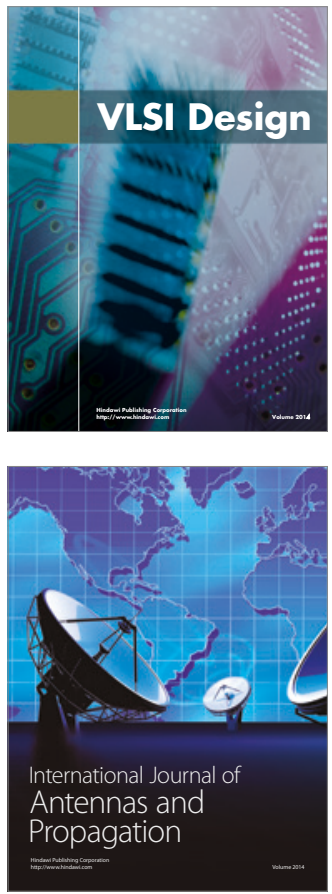

\section{Rotating}

Machinery
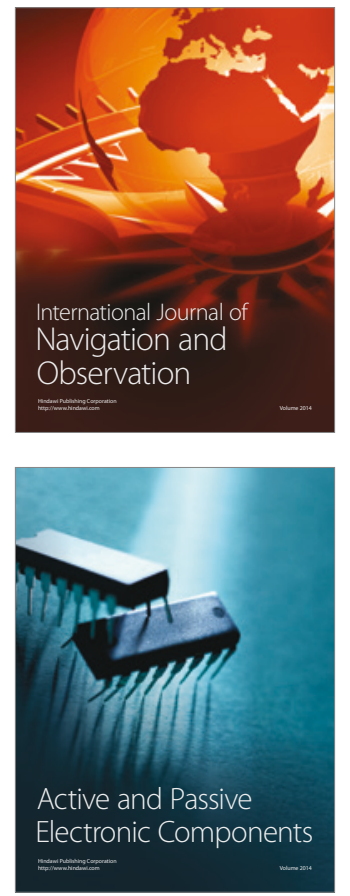
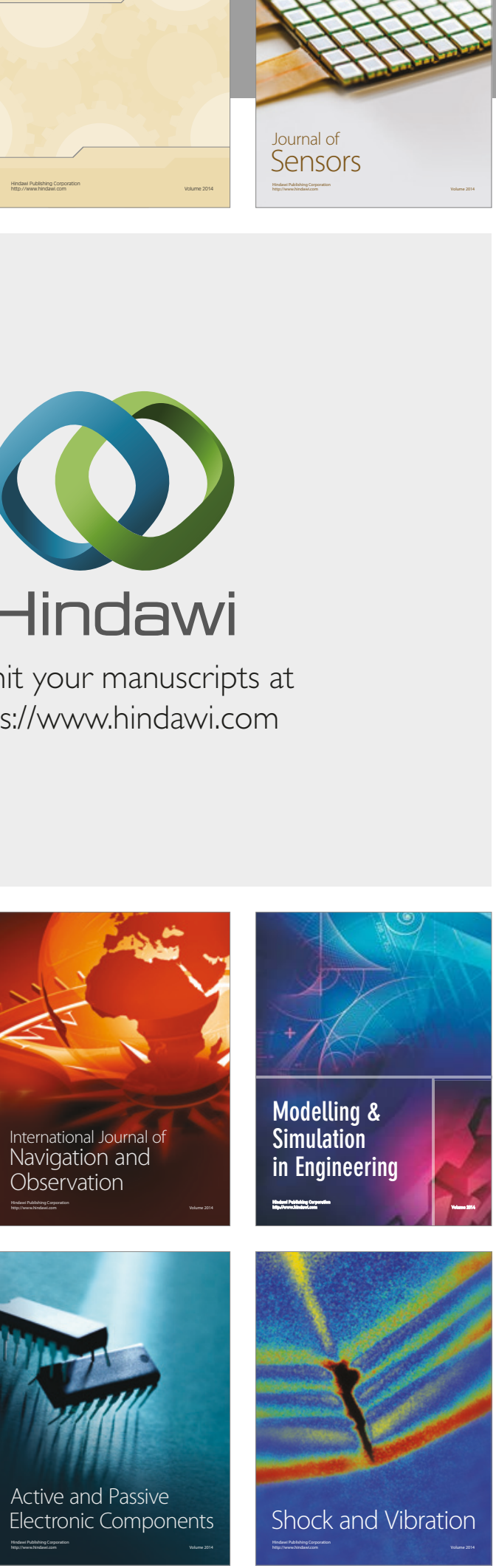
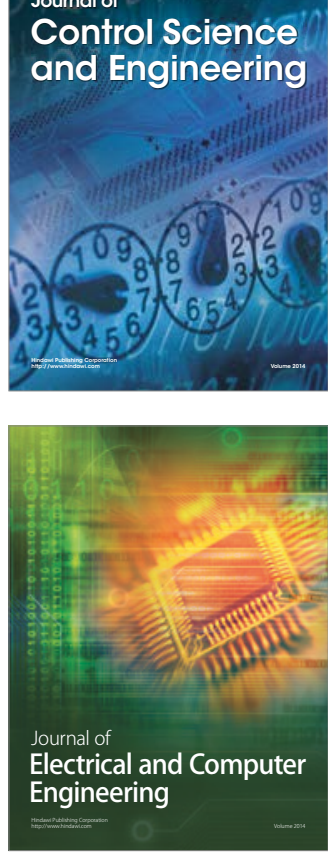

Distributed

Journal of

Control Science

and Engineering
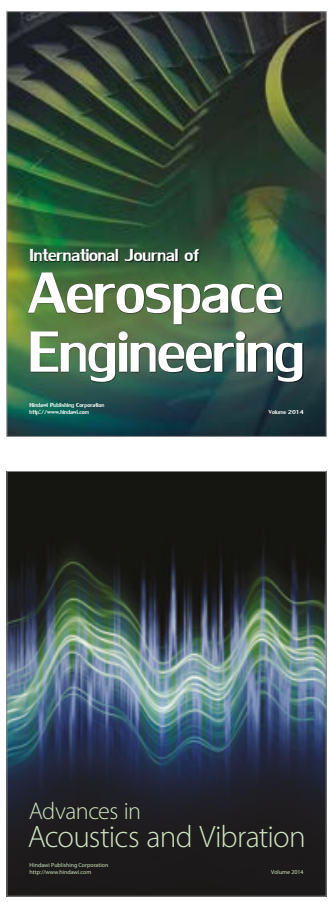

Sensor Networks 